\title{
Prolactinoma with galactorrhea and dysfunction erectile in man, a case report
}

\author{
Hari Hendarto \\ Department of Internal Medicine \\ Faculty of Medicine and Health Sciences, \\ Islamic State University (UIN) Syarif Hidayatullah Jakarta \\ Jakarta, Indonesia
}

\begin{abstract}
Prolactinoma, is the most common type of pituitary adenoma. Signs and symptoms are due to either the effect of excess hormone secretion or local compression. Here we report a male patient with prolactinoma presented with high prolactin level, severe headache, diffuse arthralgias, visual field impairment, erectile dysfunction, and galactorrhea. A 40-yearold married man was admitted to the hospital with severe headache, diffuse arthralgias, bitemporal hemianopsia, galactorrhoea, and erectile dysfunction. The pubic and axillary hairs appeared normal and the development of the external genital organs seemed normal. Endocrinological studies revealed hyperprolactinemia. The serum testosterone was low, while the thyroid hormone, growth hormone, and cortisol hormone levels were normal. An X-ray of his $L$ spine was concerning for decreased bone mass. CT scan Pituitary with contrast showed a large macroprolactinoma mass. The diagnosis of prolactinoma was made, and because the patient has progressive vision loss, combination therapy of surgery, typically a transphenoidal resection of the tumor and medication with cabergoline $0.5 \mathrm{mg}$ twice weekly, was suggested. Prolactinoma in patients can cause clinical symptoms as a result of mass effects and/or hyperprolactinaemia. Careful history and physical examinations should reveal the diagnosis.
\end{abstract}

Keywords - prolactinoma, galactorrhea, dysfunction erectile.

\section{INTRODUCTION}

Prolactinomas or prolactin-secreting adenoma, are adenomas derived from lactotroph cells in the pituitary gland, and are characterized by hypersecretion of prolactin [1]. Prolactinoma, is the most common type of pituitary adenoma that expresses and secretes prolactin to variable degrees [2]. Prolactinomas are almost always benign; malignant tumors are extremely rare. Prolactinomas are generally classified according to size as microadenomas (less than $10 \mathrm{~mm}$ in diameter), macroadenomas (more than $10 \mathrm{~mm}$ in diameter) and giant adenomas (more than $40 \mathrm{~mm}$ ) [2,3]. Signs and symptoms are erectile dysfunction, galactorrhea, persistent headache, dizziness, and even vision loss, but not all patients with pituitary tumors develop these symptoms because most tumors remain small and most do not secrete hormones in excessive amounts [2,3]. Age prevalence varies widely. Prolactinomas have been reported in patients from 2 to 80 years and is more common in women [2]. The larger the size of the prolactinoma, the greater the prolactin level and the higher the likelihood of mass-effect symptoms. Here we report a male patient with prolactinoma presented with high prolactin level, with complaints of severe headache, diffuse arthralgias, visual field impairment, erectile dysfunction, and galactorrhea.

\section{CASE SUMMARY}

A 40-year-old married man was admitted to the hospital with an episode of severe headache, diffuse arthralgias, and bitemporal hemianopsia. Furthermore, on admission, bilateral expressible galactorrhoea and erectile dysfunction was present. The pubic and axillary hairs appeared normal, and the development of the external genital organs seemed normal. Endocrinological studies revealed hyperprolactinemia of 42.000 (Ref: 4.04-15.20) ng/ml. The serum testosterone was low, while the thyroid hormone, growth hormone, and cortisol hormone levels were normal. An X-ray of his L spine was concerning for decreased bone mass. CT scan Pituitary with contrast showed a large macroprolactinoma mass. The diagnosis of prolactinoma was made, and because the patient has progressive vision loss, combination therapy of surgery, typically a transphenoidal resection of the tumor and medication with cabergoline $0.5 \mathrm{mg}$ twice weekly, was suggested.

\section{DISCUSSION}

Pituitary tumors, the most common pathologic cause of galactorrhea, can result in hyperprolactinemia by producing prolactin or blocking the passage of dopamine from the hypothalamus to the pituitary gland [4]. Prolactin is normally inhibited by the neurotransmitter dopamine coming from the hypothalamus, so that hypothalamic or pituitary stalk disease may cause a decrease in this inhibition and a rise in prolactin levels [3]. Many medications that block dopamine receptors can also cause prolactin elevations [1]. Patients with chronic hypothyroidism may have pituitary enlargement related to chronically subsequent hypersecretion of TSH and thyrotroph cell hyperplasia. 
The pituitary hyperplasia could be mistaken for prolactinoma, and patients could undergo unnecessary operation [4]. Therefore, it is important to check the function of thyroid gland in all patients with hyperprolactinaemia or in patients with pituitary enlargement before the diagnosis of prolactinoma is made. The presentation of our patient showed normal thyroid hormone level, and without any significant past medical history that block dopamine receptors.

In very large tumors, compression of pituitary cells or of the hypothalamic-pituitary stalk can cause neurological symptoms which include headaches, as found in this patient. In patients with prolactinomas, underlying mechanisms leading to headache remain uncertain, however headaches associated with hyperprolactinemia is responsive to dopamine agonists [5]. Previous studies indicate that prolactinomas may be present with headaches, similar to migraines, thus measurements of prolactin levels should be considered in patients presented with trigeminal autonomic cephalalgias [5]. About $80 \%$ of prolactinomas in men are large tumors which can cause space-occupying lesions with compressions of the optic chiasm. Visual impairment can range from quadrantanopia to classical bitemporal hemianopsia. [2].

In man, elevated prolactin levels suppress the hypothalamic-pituitary-gonadal axis, resulting in hypogonadism, which may include low testosterone levels leading to erectile dysfunction, decreased libido, and impotence which can be quite distressing for the patient [3]. Studies on bone loss have generally focused on postmenopausal women or hyperparathyroidism [6]. However, long term hypogonadism may also lead to reduced bone mineral density due to the inhibitory effect of prolactin on sex steroids [7]. This hypothesis is supported by a study that shows that when testosterone levels are restored to within normal limits, irrespective of prolactin levels, there is improvement in bone mineral density [8].

The diagnosis of prolactinoma requires both radiographic evidence of pituitary adenoma and laboratory analyses of hyperprolactinaemia. MRI will determine whether a tumor is present and its size. More than $90 \%$ of prolactinomas are microadenomas [3]. Normal prolactin levels in men are below $20 \mathrm{ng} / \mathrm{dl}$ [2]. In general, serum prolactin levels parallel with tumor size. Prolactin values between the normal and $100 \mathrm{ng} / \mathrm{ml}$ may be due to psychoactive drugs or microadenomas. Most of the time, patients with prolactin levels over $250 \mathrm{ng} / \mathrm{ml}$ will have macroadenomas [2]. Non prolactin producing macroadenomas can cause prolactin elevations from disinhibition of prolactin secretion by compressing the pituitary stalk or hypothalamus, however, when prolactin levels are more than $200 \mathrm{ng} / \mathrm{ml}$, it is almost always due to adenoma rather than stalk compression [2]. If the tumor is very large, the very high prolactin levels may rarely saturate the antibodies in some assays, leading to artificially low or normal results (hook effect). In this case, prolactin levels should be rerun at 1:100 dilution to exclude this [3]. The pathogenesis of most pituitary adenomas remains unknown, therefore, genetic evaluation is not warranted for patients presented with adenomas unless there is a compatible family history [3]. The goals of treatment for this patient, are to normalize prolactin levels and ameliorate its clinical consequences, allowing restoration of sexual dysfunction and reduce tumor size to relieve visual defect. Pharmacological therapy with dopamine agonists (bromocriptine and cabergoline) is the first line of treatment for prolactinomas due to its effectiveness in normalizing prolactin levels [7]. Dopamine agonist therapy has also shown marked reductions in tumor size via mechanisms that are not yet fully understood. However, patients should have a careful evaluation for hypopituitarism and assessment for visual field defects. Visual field defects in this patient requires urgent surgery to preserve visual function. Even though prolactinomas respond well to dopamine agonists, in this patient, testing shows abnormal visual fields, thus consideration was given to surgery. Transsphenoidal selective adenoma resection, because the patient has progressive vision loss, and combination therapy of surgery, typically a transphenoidal resection of the tumor and medication with cabergoline $0.5 \mathrm{mg}$ twice weekly, was suggested $[3,5]$.

Transsphenoidal surgery is a therapeutic option and can achieve a normalization of prolactin in $65 \%$ to $85 \%$ of patients with microadenomas and $30 \%$ to $40 \%$ with macroadenomas, with recurrence rates of $20 \%$ over 10 years [3]. The overall mortality rate following transsphenoidal surgery is less than $0.5 \%$ [1]. Radiotherapy as primary therapy is rarely done and generally reserved for patients who do not achieve adequate reduction in tumor size, hormone levels, or both in response to surgery, medical therapy, or both $[1,3]$.

\section{CONCLUSION}

Prolactinoma in patients can cause clinical symptoms as a result of mass effect and/or hyperprolactinaemia. Careful history and physical examinations should reveal the diagnosis.

\section{REFERENCES}

[1] M. Kars, O.M. Dekkers, A.M. Pereira, J.A Romijin, "Update in prolactinomas," Neth. J. Med. vol.68, pp.104-112, 2010.

[2] F.F. Casanueva, M.E. Molitch, J.A. Schlechte, R. Abs, M.D. Bronstein, T. Brue, et al, "Guidelines of the Pituitary Society for the diagnosis and management of prolactinomas," Clin. Endocrinol. vol.65, Oxford, pp.265-273, 2006

[3] M.E Molitch, "Diagnosis and Treatment of Pituitary Adenomas. A Review,"JAMA. vol.317, pp.516-524, 2017.

[4] A.K.C. Leung, D. Pacaud, "Diagnosis and Management of Galactorrhea," AFP. vol.70, pp.543-550, 2004.

[5] M. Kallestrup, H. Kasch, T. Østerby, E. Nielsen, S. Troels, T. Jensen, J.O. Jørgensen, "Prolactinoma-associated headache and dopamine agonist treatment," Cephalalgia. vol.34, pp.493-502, 2014.

[6] H. Hendarto H, "Parathyroid Adenoma in a Young Female Presenting Multiple Fractures and Postoperative Hungry Bone Syndrome," Acta. Med. Indones. vol.49, pp.69-73, 2017.

[7] A. Wong, J.A. Eloy, W.T. Couldwell, J.K. Liu, "Update on prolactinomas. Part 2: Treatment and management strategies," J. Clin Neurosci. vol.22, pp.1568-1774, 2015.

[8] S. Sperling, H. Bhatt, "Prolactinoma: A Massive Effect on Bone Mineral Density in a Young Patient," Case Reports in Endocrinology, pp.1-3, 2016. 